This item was submitted to Loughborough's Research Repository by the author.

Items in Figshare are protected by copyright, with all rights reserved, unless otherwise indicated.

\title{
Evaporation of pinned sessile microdroplets of water on a highly heat- conductive substrate: Computer simulations
}

PLEASE CITE THE PUBLISHED VERSION

http://dx.doi.org/10.1140/epjst/e2013-01789-y

\section{PUBLISHER}

(c) EDP Sciences. Published by Springer

\section{VERSION}

AM (Accepted Manuscript)

\section{PUBLISHER STATEMENT}

This work is made available according to the conditions of the Creative Commons Attribution-NonCommercialNoDerivatives 4.0 International (CC BY-NC-ND 4.0) licence. Full details of this licence are available at: https://creativecommons.org/licenses/by-nc-nd/4.0/

\section{LICENCE}

CC BY-NC-ND 4.0

\section{REPOSITORY RECORD}

Semenov, Sergey, Victor Starov, and Ramon Rubio. 2017. "Evaporation of Pinned Sessile Microdroplets of Water on a Highly Heat-conductive Substrate: Computer Simulations". figshare. https://hdl.handle.net/2134/25799. 


\title{
Evaporation of pinned sessile microdroplets of water on a
}

\section{highly heat-conductive substrate: computer simulations}

\author{
Sergey Semenov ${ }^{1,}$ a, Victor M. Starov ${ }^{1, b}$, Ramon G. Rubio ${ }^{2, ~ c ~}$ \\ ${ }^{1}$ Dept. of Chemical Engineering, Loughborough University, LE11 3TU Loughborough, UK \\ ${ }^{2}$ Dept. of Química Física I, Universidad Complutense, 28040 Madrid, Spain \\ a e-mail: s.semenov@lboro.ac.uk \\ b e-mail: v.m.starov@lboro.ac.uk \\ c e-mail: rgrubio@quim.ucm.es
}

\begin{abstract}
The aim of current numerical study is to investigate the influence of individual effects (kinetic effects, latent heat of vaporization, Marangoni convection, Stefan flow, droplet's surface curvature) on the rate of evaporation of water droplet placed on a highly heat conductive substrate for different sizes of the droplet (down to submicron sizes). We performed simulations for one particular set of parameters: ambient relative air humidity is set to $70 \%$, ambient temperature is $20^{\circ} \mathrm{C}$, contact angle is $90^{\circ}$, and substrate material is copper. Suggested model combines both diffusive and kinetic models of evaporation. The obtained results allow estimating the characteristic droplet sizes where each of the mentioned above phenomena become important or can be neglected.
\end{abstract}

KEYWORDS: sessile droplet, evaporation, kinetic effects

\section{Introduction}

The evaporation of sessile liquid droplets is important in variety of industrial applications such as spray cooling [1, 2], ink-jet printing [3], tissue engineering [4], printing of microelectromechanical systems (MEMS) [5], surface modification [6, 7], various coating processes [8], as well as biological 
applications [9]. A number of theoretical and experimental investigations have been focussed on investigations of this phenomenon [10 - 18].

Studying the evaporation of microdroplets can help understanding the influence of Derjaguin's (disjoining/conjoining) pressure acting in a vicinity of the apparent three-phase contact line [19 - 21].

The aim of the presented computer simulations is to show how the evaporation of pinned sessile submicron droplets of water on a solid surface differs from the evaporation of millimetre size droplets. The obtained results prove the importance of kinetic effects, whose influence becomes more pronounced for submicron droplets.

The model used below includes both diffusive and kinetic models of evaporation simultaneously. Hertz-Knudsen-Langmuir equation [22, 23] is used as a boundary condition at the liquid-gas interface instead of a condition of saturated vapour. The overall evaporation rate is limited either by the rate of vapour diffusion into ambient gas or by the rate of molecules transfer across the liquid-gas interface. As a result the vapour concentration at the liquid-gas interface falls in between its saturated value and its value in ambient gas. This intermediate value of vapour concentration at the liquid-gas interface drives both transition of molecules from liquid to gas (kinetic flux) and vapour diffusion into ambient gas (diffusive flux). Thus the rate of evaporation is limited by the slower of these two processes.

Computer simulations are performed using the software COMSOL Multiphysics. The dependence of total molar evaporation flux, $J_{c}$, on the radius of the contact line, $L$, of pinned droplets is studied below using a quasi-steady state approximation.

\section{Problem statement}

Below only droplets of size less than $1 \mathrm{~mm}$ are under consideration. That is the influence of gravity is neglected. It is assumed that axisymmetric sessile droplet forms a sharp three-phase contact line and maintains a spherical-cap shape of the liquid-gas interface due to the action of liquid-gas interfacial tension. Geometry of the problem is schematically presented in Fig. 1. 


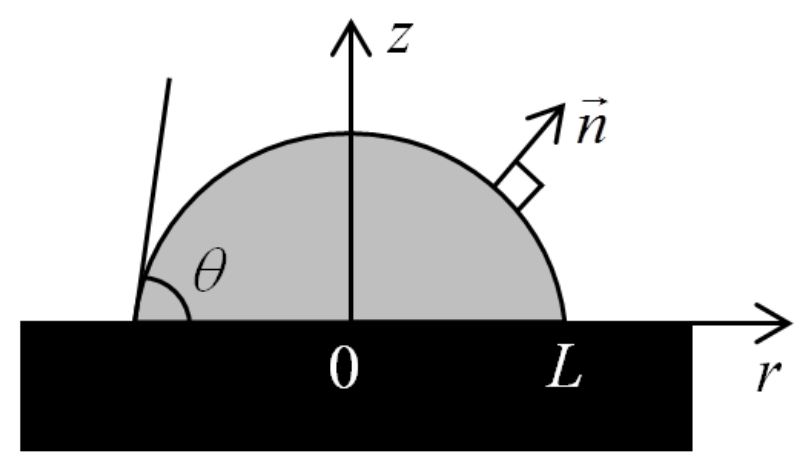

Fig. 1. An axisymmetric sessile droplet on a solid substrate. $\theta$ and $L$ are contact angle and radius of the droplet base. In present studies the contact angle is $90^{\circ}$.

Due to small size of the evaporating droplets under consideration, the diffusion of vapour in the gas phase dominates its convective transport. The latter is confirmed by small values of both thermal and diffusive Peclet numbers: $\mathrm{Pe}_{\kappa}=L u / \kappa<0.05$; $\mathrm{Pe}_{D}=L u / D<0.04$, where $L$ is the radius of the contact line, $u(<1 \mathrm{~mm} / \mathrm{s})$ is the characteristic velocity of vapour convection due to evaporation (Stefan flow) and Marangoni convection, $\kappa$ is the thermal diffusivity of the surrounding air, and $D$ is the diffusion coefficient of vapour in air at standard conditions.

As in Ref. [24] the problem is solved under a quasi-steady state approximation. That is, all time derivatives in all equations are neglected. The quasi-steady solution of the problem gives simultaneous distribution of heat and mass fluxes in the system.

Volume of the droplet diminishes because of evaporation. Therefore the liquid-air interface moves with some velocity, which can be calculated based on the knowledge of the evaporation rate and two particular assumptions: cap of the droplet preserves its spherical shape, and contact line is pinned ( $L=$ const $)$. Regime of moving contact line is not considered in present study.

The parameters of the following materials are used in the present computer simulations: copper as the substrate, water as the liquid in a droplet, and a humid air as a surrounding medium. The pressure in the surrounding gas is equals to the atmospheric pressure, the ambient temperature is $20^{\circ} \mathrm{C}$, the ambient air humidity is $70 \%$, and the contact angle is $90^{\circ}$.

\subsection{Governing equations in the bulk phases}


This section presents the problem statement, which is based on the one, proposed by Krahl et al. [25].

The following governing equations describe the heat and mass transfer in the bulk phases:

heat transfer in a solid phase:

$$
\Delta T=0,
$$

where $\Delta$ is the Laplace operator, and $T$ is the temperature;

heat transfer inside of fluids (liquid or gas):

$$
\vec{u} \cdot \nabla T=\kappa \Delta T,
$$

where $\vec{u}$ is the fluid velocity, $\nabla$ is the gradient operator, and $\kappa$ is the thermal diffusivity of the fluid; incompressible Navier-Strokes equations are used to model hydrodynamic flows in both fluids (liquid or gas):

$$
\rho \vec{u} \cdot \nabla \vec{u}=\nabla \cdot \mathbf{T},
$$

where $\rho$ is the fluid density, $\nabla \vec{u}$ is the gradient of the velocity vector, $\mathbf{T}$ is the full stress tensor, and $\nabla \cdot \mathbf{T}$ is the dot-product of nabla operator and the full stress tensor. The full stress tensor is expressed via hydrodynamic pressure, $p$, and viscous stress tensor, $\boldsymbol{\pi}$, as:

$$
\mathbf{T}=-p \mathbf{I}+\boldsymbol{\pi},
$$

where $\mathbf{I}$ is the identity tensor. Continuity equation for fluids is also used:

$$
\nabla \cdot \vec{u}=0 .
$$

The diffusion equation for vapour in gas phase:

$$
\Delta c=0,
$$

where $c$ is the molar concentration of the liquid vapour.

\subsection{Boundary conditions}

No-slip and no-penetration boundary conditions are used for Navier-Stokes equations at the liquidsolid and gas-solid interfaces, resulting in zero velocity at these interfaces:

$$
\vec{u}=0 .
$$


Let $\Gamma$ be the liquid-gas interface. Let also $j_{c}$ be a density of a molar vapour flux across the liquid-gas interface, then a density of mass vapour flux, $j_{m}$, across this interface is $j_{m}=j_{c} \cdot M$, where $M$ is the molar mass of an evaporating substance (water). Let $u_{\Gamma}$ be the normal velocity of the liquid-gas interface itself, see Fig. 2. Then the boundary condition for the normal velocity of liquid at the liquid-gas interface reads:

$$
\rho_{l}\left(\vec{u}_{l} \cdot \vec{n}-u_{\Gamma}\right)=j_{m},
$$

where $\rho_{l}$ is the liquid density, subscript $l$ stands for liquid. Expressions for evaporation flux, $j_{m}$, and normal interfacial velocity, $u_{\Gamma}$, are specified below. A condition of the stress balance at the liquid-gas interface is used to obtain boundary conditions for the pressure and tangential velocity:

$$
(\mathbf{T} \cdot \vec{n})_{l}-(\mathbf{T} \cdot \vec{n})_{g}=-\gamma(\nabla \cdot \vec{n})_{\Gamma} \vec{n}+\gamma_{T}^{\prime}\left(\nabla_{\Gamma} T\right),
$$

where subscripts $l$ and $g$ stand for liquid and gas, respectively; $\mathbf{T}$ is the full stress tensor; $\gamma$ is the interfacial tension of the liquid-gas interface; $\gamma_{T}^{\prime}$ is the derivative of the interfacial tension with the temperature; $\nabla_{\Gamma} T$ is the surface gradient of temperature; $(\nabla \cdot \vec{n})_{\Gamma}$ is the divergence of the normal vector at the liquid-gas interface, which is equal to the curvature of the interface. Boundary condition (3) is a vector one. The boundary conditions in normal and tangential direction are deduced as follows: (i) the boundary condition for thermal Marangoni convection, which determines the tangent component of the velocity vector is obtained by multiplying Eq. (3) by the tangential vector $\vec{\tau}$ (see Fig. 2) and neglecting the viscous stress in the gas phase (due to small gas viscosity compared to the liquid viscosity); (ii) the similar procedure with normal vector $\vec{n}$ (see Fig. 2) results in a boundary condition for pressure in the liquid at the liquid-gas interface. 


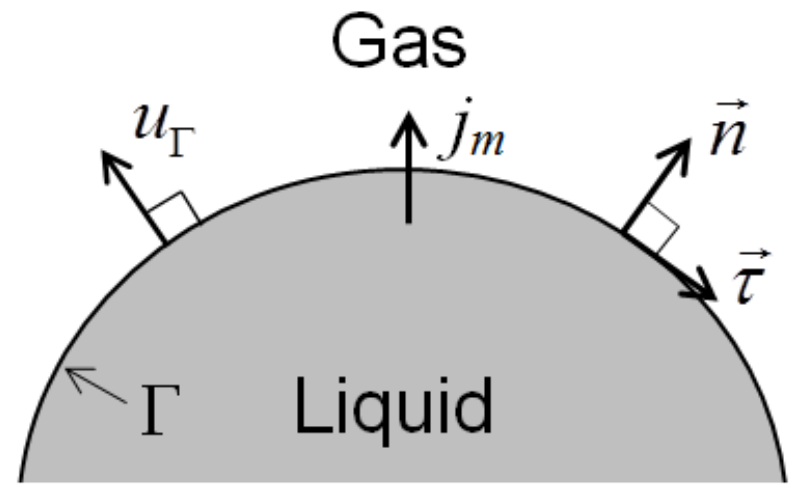

Fig. 2: Notations: $\Gamma$ is the liquid-vapour interface; $u_{\Gamma}$ is the normal velocity of the interface $\Gamma$ in the direction of the normal unit vector $\vec{n}$ (from the liquid phase to the gaseous one); $\vec{\tau}$ is the unit vector tangential to the interface $\Gamma ; j_{m}$ is the mass flux across the interface $\Gamma$.

The normal flux of vapour at the gas-solid interface is zero because there is no penetration into the solid surface:

$$
\nabla c \cdot \vec{n}=0
$$

where $c$ is the molar concentration of the vapour in the air; and $\vec{n}$ is the unit vector, perpendicular to the solid-gas interface.

Below we consider gas phase as the mixture of vapour and dry air. Note that due to mass conservation law the mass flux of vapour, $j_{m}$, perpendicular to the liquid-gas interface in the vapour phase should be equal to the mass flux of liquid perpendicular to the interface in the liquid phase:

$$
j_{m}=\rho_{l}\left(\vec{u}_{l} \cdot \vec{n}-u_{\Gamma}\right)=\rho_{v}\left(\vec{u}_{g} \cdot \vec{n}-u_{\Gamma}\right)-D_{\text {vapour in air }} \nabla \rho_{v} \cdot \vec{n} \text {. }
$$

Density of mass flux of dry air, $j_{m}$, air, across the liquid-gas interface is assumed to be zero:

$$
j_{m, \text { air }}=\rho_{\text {air }}\left(\vec{u}_{g} \cdot \vec{n}-u_{\Gamma}\right)-D_{\text {air in vapour }} \nabla \rho_{\text {air }} \cdot \vec{n}=0 \text {, }
$$

where $\rho_{v}$ and $\rho_{\text {air }}$ are densities of vapour and dry air, respectively; $D$ is the diffusion coefficient; $\vec{u}_{l}$ and $\vec{u}_{g}$ are velocity vectors of liquid and gas, respectively; $u_{\Gamma}$ and $\vec{n}$ are shown in Fig. 2 . As the gas (water vapour + dry air) under consideration includes more than one species of molecules, then the mass flux for each species in the air phase consists of two components: convective part, $\rho \vec{u}_{g}$, and diffusive 
one, $D \nabla \rho$. Flux in the pure liquid includes only a convective term, $\rho_{l} \vec{u}_{l}$. Normal fluxes at the liquidgas interface are considered relative to the liquid-gas interface. The latter results in an additional term: $-\rho u_{\Gamma}$. Let us adopt the following assumption: $\rho_{g}=\rho_{\text {air }}+\rho_{v}=$ const. The latter assumption means incompressibility of the gaseous phase. This assumption results in

$$
\nabla \rho_{\text {air }}=-\nabla \rho_{v} .
$$

A direct consequence of the above assumption is:

$$
D_{\text {air in vapour }}=D_{\text {vapour in air }}=D \text {. }
$$

After substitution of Eqs. (6) and (7) into Eqs. (4) and (5) and simple algebraic manipulations we arrive to an expression for the density of mass flux across the liquid-gas interface, $j_{m}$, as a function of molar vapour concentration, $c$, in gas phase:

$$
j_{m}=\frac{-D \nabla \rho_{v} \cdot \vec{n}}{1-\rho_{v} / \rho_{g}}=\frac{-D \nabla c \cdot \vec{n}}{1 / M-c / \rho_{g}},
$$

where the following relation has been used: $\rho_{v}=c M, M$ is the molar mass of an evaporating substance (water). Eq. (8) connects the evaporation flux, $j_{m}$, with both the gradient of vapour concentration in the normal direction and the concentration itself. On the other hand, the rate of mass transfer across the liquid-gas interface is given by the Hertz-Knudsen-Langmuir equation [22, 23]:

$$
j_{m}=\alpha_{m} \sqrt{\frac{M R T}{2 \pi}}\left(c_{\text {sat }}(T)-c\right),
$$

where $\alpha_{m}$ is the mass accommodation coefficient (probability that uptake of vapour molecules occurs upon collision of those molecules with the liquid surface); $R$ is the universal gas constant; $T$ and $c$ are the local temperature in ${ }^{\circ} \mathrm{K}$ and molar vapour concentration at the liquid-gas interface, respectively; $c_{s a t}$ is the molar concentration of saturated vapour. Molar concentration of saturated vapour is taken as a function of a local temperature and a local curvature of the liquid-gas interface according to ClausiusClapeyron [24] and Kelvin [26] equations. 
Combining Eqs. (8) and (9) we obtain a boundary condition for diffusion equation (1) at the liquid-gas interface:

$$
\frac{-D \nabla c \cdot \vec{n}}{1 / M-c / \rho_{g}}=\alpha_{m} \sqrt{\frac{M R T}{2 \pi}}\left(c_{s a t}(T)-c\right) .
$$

Summing equations (4) and (5), and taking into account the assumption $\left(\rho_{g}=\rho_{\text {air }}+\rho_{v}=\right.$ const $)$, we arrive to the boundary condition for the normal velocity of gas at the liquid-gas interface:

$$
\rho_{g}\left(\vec{u}_{g} \cdot \vec{n}-u_{\Gamma}\right)=j_{m} \text {. }
$$

Tangent velocity of gas at the liquid-gas interface is determined by the no-slip condition:

$$
\vec{u}_{g} \cdot \vec{\tau}=\vec{u}_{l} \cdot \vec{\tau}
$$

Boundary conditions of temperature continuity are applied at all interfaces (liquid-gas, liquid-solid and gas-solid):

$$
T_{l}=T_{g}, \quad T_{l}=T_{s}, \quad T_{g}=T_{s},
$$

where subscripts $l, g$ and $s$ stand for liquid, gas and solid, respectively. Continuity of the heat flux is applied on solid-liquid and solid-gas interfaces:

$$
\begin{aligned}
& -k_{l}(\nabla T)_{l} \cdot \vec{n}+k_{s}(\nabla T)_{s} \cdot \vec{n}=0, \\
& -k_{g}(\nabla T)_{g} \cdot \vec{n}+k_{s}(\nabla T)_{s} \cdot \vec{n}=0,
\end{aligned}
$$

where $k$ is the thermal conductivity of the corresponding phase; $\vec{n}$ is the unit vector, perpendicular to a corresponding interface. Note: at all these interfaces the convective heat flux is zero due to no penetration conditions. At the liquid-gas interface heat flux experiences discontinuity caused by the latent heat of vaporization and also there is a convective heat flux through this interface:

$$
\left\lfloor\rho_{l} c_{p l} T\left(\vec{u}_{l} \cdot \vec{n}-u_{\Gamma}\right)-k_{l}(\nabla T)_{l} \cdot \vec{n}\right\rfloor-\left\lfloor\rho_{g} c_{p g} T\left(\vec{u}_{g} \cdot \vec{n}-u_{\Gamma}\right)-k_{g}(\nabla T)_{g} \cdot \vec{n}\right\rfloor=j_{c} \Lambda,
$$

where $c_{p l}$ and $c_{p g}$ are specific heat capacities at constant pressure for the liquid and the gas, respectively; $\Lambda$ is the latent heat of vaporization (or enthalpy of vaporization [27], units: $\mathrm{J} / \mathrm{mol}$ ); $\vec{n}$ is the unit vector, normal to the liquid-gas interface, and pointing into the gas phase; $j_{c}$ is the surface 
density of the molar flux of evaporation $\left(\mathrm{mol} \cdot \mathrm{s}^{-1} \cdot \mathrm{m}^{-2}\right.$ ) at the liquid-gas interface. Using Eqs. (2), (10), and relation between molar and mass fluxes, $j_{m}=j_{c} \cdot M$, we derive the following expression:

$$
\rho_{l} c_{p l} T\left(\vec{u}_{l} \cdot \vec{n}-u_{\Gamma}\right)-\rho_{g} c_{p g} T\left(\vec{u}_{g} \cdot \vec{n}-u_{\Gamma}\right)=c_{p l} T j_{m}-c_{p g} T j_{m}=j_{c} M T\left(c_{p l}-c_{p g}\right) .
$$

Eq. (11) using the latter expression transforms into the required boundary condition for the heat flux discontinuity at the liquid-gas interface:

$$
-k_{l}(\nabla T)_{l} \cdot \vec{n}+k_{g}(\nabla T)_{g} \cdot \vec{n}=j_{c}\left(\Lambda-M T\left(c_{p l}-c_{p g}\right)\right) .
$$

At the axis of symmetry $(r=0)$ the following boundary conditions are satisfied:

$$
\left.\frac{\partial c}{\partial r}\right|_{r=0}=0,\left.\frac{\partial T}{\partial r}\right|_{r=0}=0,\left.\frac{\partial u_{z}}{\partial r}\right|_{r=0}=0,\left.u_{r}\right|_{r=0}=0,\left.\frac{\partial p}{\partial r}\right|_{r=0}=0,
$$

where $u_{r}$ and $u_{z}$ are radial and vertical components of the velocity vector, and $p$ is the hydrodynamic pressure.

At the outer boundary of the computational domain values of temperature, $T_{\infty}$, and vapour concentration, $c_{\infty}$, are imposed. In the case when gas convection (Stefan flow) is taken into account, condition of open boundary (zero normal stress) is imposed at the outer boundary of the gas domain:

$$
\mathbf{T} \cdot \vec{n}=0,
$$

where $\mathbf{T}$ is the full stress tensor, and $\vec{n}$ is the unit normal vector. This boundary condition allows the gas both enter and leave the domain.

In our computer simulations we assume that the droplet under consideration retains a spherical-cap shape in the course of evaporation, and contact line is pinned ( $L=$ const). Then knowing the total mass evaporation flux, $J_{m}=\int_{\Gamma} j_{m} d A$ ( $d A$ is the element of area of the interface $\Gamma$ ), we can calculate the normal velocity of the liquid-gas interface, $u_{\Gamma}$, at any point of the interface:

$$
u_{\Gamma}=\frac{-J_{m}}{\pi \rho_{l} L^{2}} \cdot \frac{z}{(z+\delta z) \cdot n_{z}+r \cdot n_{r}} \cdot \frac{1+\cos \theta}{1-\cos \theta},
$$


where $\delta \mathrm{z}=L \cdot \cos \theta / \sin \theta ; \theta$ is the contact angle; $n_{r}$ and $n_{z}$ are radial and vertical components of the vector $\vec{n}$, respectively, shown in Fig. 2; the origin of cylindrical coordinates $(r, z)$ is supposed to be at the point of intersection of axis of droplet symmetry with the liquid-solid interface (Fig. 2).

\section{Computer simulations}

Computer simulations are performed using commercial software COMSOL Multiphysics. The numerical method used in COMSOL is a finite element method with quadratic Lagrangian elements. The software transforms all equations into their weak form before discretization. The method of Lagrange multipliers is used to apply boundary conditions as constraints.

The computational domain is selected as a circle in $(r, z)$ coordinates of the cylindrical system of coordinates. The centre of this circle located at the origin of the system of coordinates. The radius of the computational domain is 100 times bigger than the radius of the contact line, $L$, which prevents the influence of the proximity of outer boundaries on the solution inside of the droplet.

The choice of contact angle $90^{\circ}$ allows avoiding the problem of singularity of diffusive evaporation flux at the three-phase contact line; but the problem of viscous stress singularity remains. Current model does not employ any physical mechanisms to overcome this problem. Instead, the mesh is refined around the contact line to reduce the area of singularity influence. Thus the size of computational mesh elements around the three-phase contact line is chosen to be 100 times smaller than the droplet size.

The growth rate for mesh elements is less than 1.1 in the whole computational domain.

\section{Results and discussion}

\subsection{Isothermal evaporation}

The model described here is an extension of the previous one [18, 24], which was developed for diffusion limited evaporation of water droplets. In distinction to the previous model, the present one takes into account additional phenomena: Stefan flow in gas, effect of curvature of the droplet's surface on saturated vapour pressure (Kelvin's equation), and kinetic effects (also known as Knudsen effects). A numerical model allows switching individual phenomena on/off in order to understand their contribution to the overall process of heat and mass transfer in the course of evaporation. 
The value of mass accommodation coefficient $\alpha_{m}$ is taken as 0.5 , which is the average experimentally measured value of $\alpha_{m}$ for water according to Ref. [22].

When Kelvin's and kinetic effects are switched off, then the model represents the case of diffusion limited evaporation. This allows us to validate the present model against the previous one [18, 24], which in turn was validated against available experimental data [18] for diffusion limited case. Computer simulations showed that there is agreement with the previous results [24] (see Fig. 3).

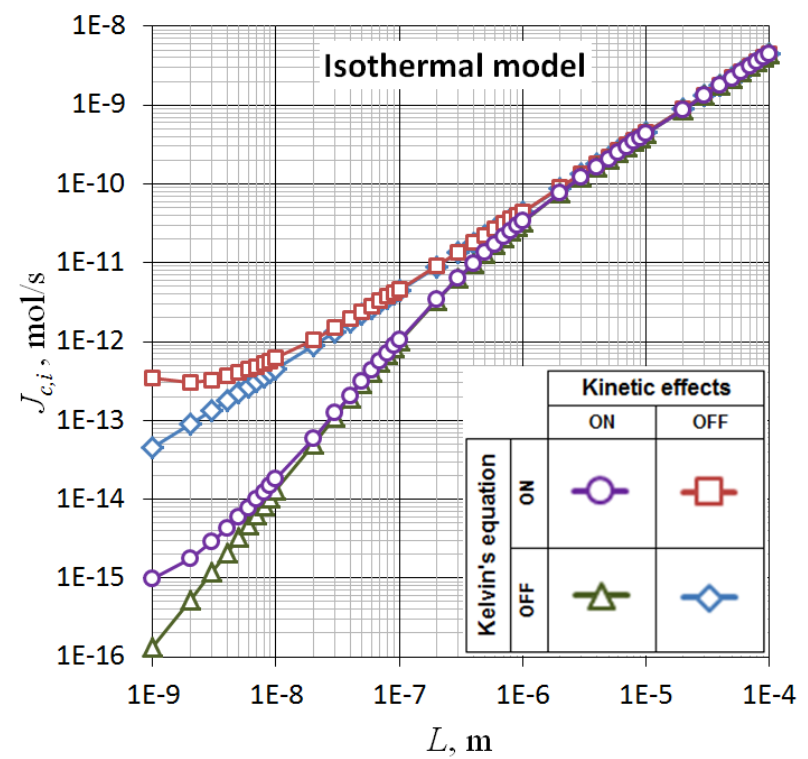

Fig. 3. Dependence of the droplet's molar evaporation flux, $J_{c, i}$, on the droplet size, $L$, for isothermal model of evaporation. Parameters used: $\alpha_{m}=0.5 ; \theta=90^{\circ}$; relative air humidity is $70 \%$. Note: results for $L<10^{-7} \mathrm{~m}$ do not have physical meaning, as additional surface forces must be included into the model. These points are shown to demonstrate the trends of curves.

In Fig. 3 the total molar fluxes of droplet's evaporation, $J_{c, i}$, are presented for various isothermal cases (index $i$ stands for the “isothermal”). The flux was computed according to the presented above model, in which the Stefan flow, heat transfer and the thermal Marangoni convection were omitted. Fig. 3 shows that kinetic effects change the slope of curves (see triangles and circles) for submicron droplets ( $\left.L<10^{-6} \mathrm{~m}\right)$ only. The influence of curvature of the droplet's surface (Kelvin's equation) becomes 
significant only for droplet sizes less than $10^{-7} \mathrm{~m}$. However, at such low sizes the surface forces action (disjoining/conjoining pressure) has to be taken into account (not included in the present model).

Note once more that the presented model is valid only for droplet size bigger than the radius of surface forces action, which is around $10^{-7} \mathrm{~m}=0.1 \mu \mathrm{m}$. That is, the data in Fig. 3 for the droplet size smaller than $10^{-7} \mathrm{~m}$ are presented only to show the trend. Fig. 3 shows that if the radius of the droplet base bigger than $10^{-7} \mathrm{~m}$ then (i) deviation of the saturated vapour pressure caused by the droplet curvature (Kelvin’s equation) can be neglected, (ii) a deviation from the pure diffusion model of evaporation can be neglected for the droplet size bigger than $10^{-6} \mathrm{~m}$, (iii) this deviation becomes noticeable only if the droplet size is less than $10^{-6} \mathrm{~m}$. This deviation is caused by an increasing influence of the kinetic effects at the liquid-gas interface (Hertz-Knudsen-Langmuir equation) and this theory should be applied together with the diffusion equation of vapour in the air if the droplet size is less than $10^{-6} \mathrm{~m}$.

The latter conclusions show that a consideration of evaporation of microdroplets, completely covered by the surface forces action (that is less than $10^{-7} \mathrm{~m}$ ), should take into account both deviation of the saturated vapour pressure caused by the droplet curvature and the kinetic effects.

According to the model of diffusion limited evaporation the evaporation flux, $J_{c, i}$, must be linearly proportional to the droplet size, $L$, that is $J_{c, i} \sim L$. The latter is in agreement with data presented in Fig. 3 for droplets bigger than $10^{-6} \mathrm{~m}$. However, for a pure kinetic model of evaporation (no vapour diffusion, uniform vapour pressure in the gas) flux $J_{c, i}$ is supposed to be proportional to the area of the droplet's surface, that is in the case of pinned droplets (constant contact area) $J_{c, i} \sim L^{2}$ should be satisfied. To check the validity of the latter models at various droplet sizes let us assume that the dependency of the evaporation flux on the droplet radius has the following form $J_{c, i}=A(\theta) \cdot L^{n}$, where $n$ is the exponent to be extracted from our model and $A$ is a function of the contact angle, $\theta$. In general it is necessary to calculate the partial derivative

$$
n=\partial\left(\ln J_{c, i}\right) / \partial(\ln L)
$$


in order to compute the exponent $n$ using the present model. However, in the case of pinned droplet the only varying parameter for each individual curve in Fig. 3 was the contact line radius, $L$. Hence, the partial derivatives in Eq. (12) can be replaced by ordinary derivatives and in this way $n$ was calculated using data presented in Fig. 3. The calculated values of $n$ are presented in Fig. 4.

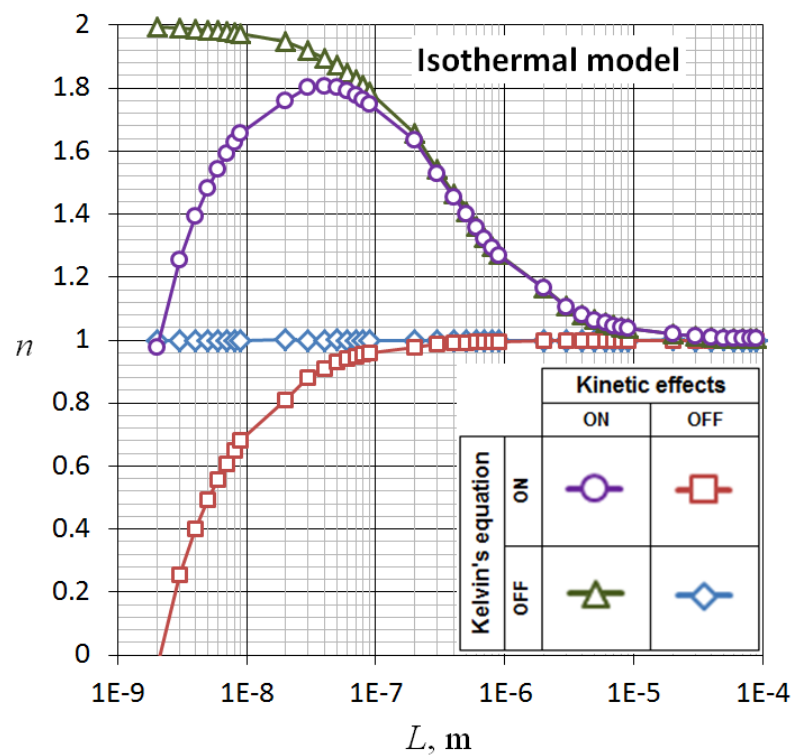

Fig. 4. Exponent $n$ for the dependence $J_{c, i}=A(\theta) \cdot L^{n}$ for the isothermal model of evaporation. Parameters used: $\alpha_{m}=0.5 ; \theta=90^{\circ}$; relative air humidity is $70 \%$. Note: results for $L<10^{-7} \mathrm{~m}$ do not have physical meaning, as surface forces action must be included into the model here. These points are shown to demonstrate the trends of curves.

One can see from Fig. 4 that the exponent $n$, as expected, is equal to 1 for a pure diffusive isothermal model of evaporation within the whole studied range of $L$ values (diamonds in Fig. 4). In the case when kinetics effects and Kelvin's equation are both taken into account additional to the pure diffusion Fig. 4 shows that the diffusion model of evaporation dominates for droplets with the size bigger than $10^{-5} \mathrm{~m}$, that is for droplets bigger than $10 \mu \mathrm{m}$.

Taking into account kinetic effects only additional to the diffusion without Kelvin's equation (triangles in Fig. 4) results in a smooth transition from the linear dependence $J_{c, i} \sim L$, that is $n=1$ (diffusive model) to the quadratic one $J_{c, i} \sim L^{2}$, that is $n=2$ (kinetic model) as the size of the droplet decreases down to $L=10^{-9} \mathrm{~m}$ (see Fig. 4). The latter shows that $J_{c, i}$ is tending to be proportional to $L^{2}$ as 
the size of the droplet decreases, which means that evaporation flux becomes proportional to the area of the liquid-gas interface. Influence of the curvature (Kelvin's equation) on the saturated vapour pressure results in a substantially lower exponent $n$ as compared with the kinetic theory (Fig. 4). However, the latter happens only for a droplet completely in the range of surface forces action, that is, less than $10^{-7} \mathrm{~m}$ [20]. Below this limit the droplet does not have a spherical cap shape any more even on the droplet's top (microdroplets according to Ref. [20]). Evaporation process in the latter case should be substantially different from the considered above. Thus the range of sizes less than $10^{-7} \mathrm{~m}$ is not covered by the presented theory.

\subsection{Influence of thermal effects}

Computer simulations were also performed including both Kelvin’s equation and kinetic effects in the case when the thermal effects were taken into account. The latter was made to show the influence of the latent heat of vaporization, Marangoni convection and Stefan flow on the evaporation process. Droplet’s evaporation rates, $J_{c}$, were normalized using those, $J_{c, i}$ (circles in Fig. 3), from the isothermal model. Results are presented in Fig. 5.

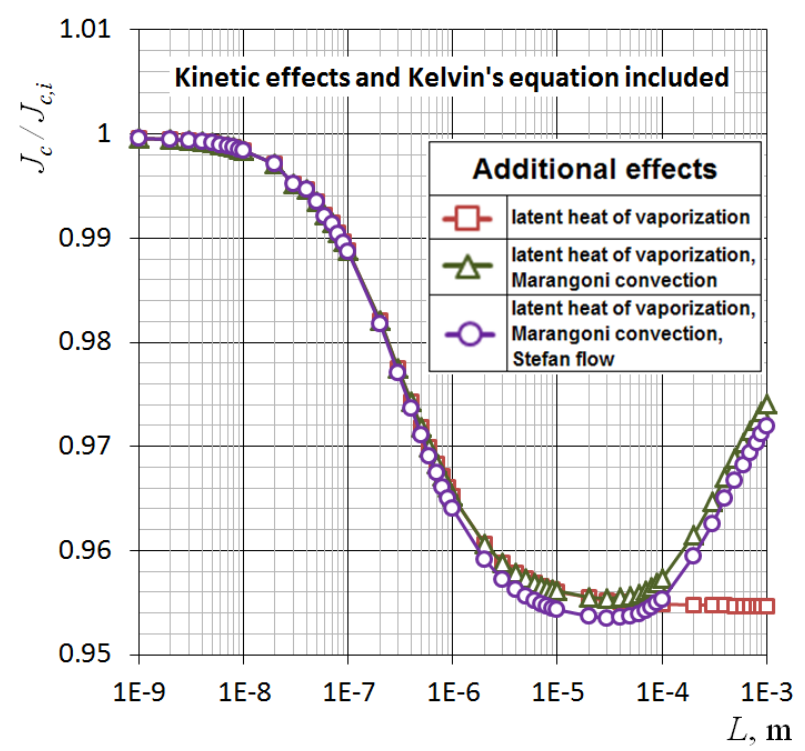

Fig. 5: Influence of latent heat of vaporization, Marangoni convection, and Stefan flow on the evaporation rate in the case when kinetic effects and Kelvin's equation are included into the model. $J_{c}$ and $J_{c, i}$ are total molar flux of evaporation and the one in the isothermal case, respectively. 
Fig. 5 shows that latent heat of vaporization reduces the evaporation flux as compared to the isothermal case (that is $J_{C} / J_{c, i}<1$ ) in all cases considered. The reason is a temperature decrease at the droplet's surface due to heat consumption during the evaporation process. This reduces the value of the saturated vapour pressure at the droplet's surface and, subsequently, the rate of vapour diffusion into the ambient gas. The relative reduction of the evaporation rate (caused by the latent heat of vaporization) reaches the maximum for droplets with $L \sim 10^{-5} \mathrm{~m}$. The latter size according to Figs. 3 and 4 is in the range of diffusion limited evaporation. For smaller droplets, when the kinetic effects come into play, the influence of the latent heat on evaporation rate, $J_{c}$, decreases. The reason for that is that vapour above the droplet surface according to the kinetic model of evaporation is not saturated and therefore its pressure is less influenced by the local temperature but more influenced by the relative humidity of the ambient air. If we exclude kinetic effects and Kelvin's equation from both non-isothermal $\left(J_{c}\right)$ and isothermal $\left(J_{c, i}\right)$ models, and include only latent heat of vaporization, then the ratio $J_{c} / J_{c, i}$ becomes independent on $L$ and equal its value as for diffusion limited case (millimetre sized droplets).

Taking into account kinetic effects, Kelvin's equation, latent heat of vaporization, and thermal Marangoni convection (for water droplets) affects droplets of size $L>10^{-5} \mathrm{~m}$ (triangles in Fig. 5). In this case evaporation rate, $J_{c}$, is lower than that for isothermal model and higher than that if the latent heat included but without Marangoni convection (squares in Fig. 5).

For water droplets of size $L<10^{-5} \mathrm{~m}$ the influence of Marangoni convection is negligible and evaporation rate, $J_{c}$, coincides with the one for a model which includes only latent heat of vaporization (squares in Fig. 5).

The effect of Stefan flow in surrounding gas slightly changes the evaporation rate in the present model (circles in Fig. 5) and makes it lower due to an appearance of an outward convective heat flux in the gas above the droplet. This reduces the heat flux from the ambient environment to the droplet's surface through the gas phase. Thus temperature of the droplet's surface becomes lower, which reduces the evaporation rate. In our particular case this effect appeared to be much weaker than the effects of latent heat of vaporization. Stefan flow effect is also weaker than the effect of thermal Marangoni convection 
for $L>10^{-4} \mathrm{~m}$, but a bit stronger for $L<10^{-4} \mathrm{~m}$. However, in any case the influence of the Stefan flow is small and can be neglected.

Note that the influence of the thermal effects on the kinetics of evaporation is less than 5\% (according to Fig. 5).

\section{Conclusions}

Computer simulations of evaporation of small sessile droplets of water are performed. Present model combines diffusive and kinetic models of evaporation. The effect of latent heat of vaporization, thermal Marangoni convection and Stefan flow in the surrounding gas were investigated for a particular system: water droplet on a heat conductive substrate (copper) in air at standard conditions. Results of modelling allow estimating the characteristic droplet sizes when each of the mentioned above phenomena become important or can be neglected.

The presented model is valid only for droplet size bigger than the radius of surface forces action, which is around $10^{-7} \mathrm{~m}=0.1 \mu \mathrm{m}$. That is, the data in Figs. $3-5$ for the droplet size smaller than $10^{-7} \mathrm{~m}$ are presented only to show the trend. When the radius of the droplet base, $L$, bigger than $10^{-7} \mathrm{~m}$ then (i) deviation of the saturated vapour pressure caused by the droplet curvature (Kelvin's equation) can be neglected, (ii) a deviation from the pure diffusion model of evaporation can be neglected for the droplet

size bigger than $10^{-6} \mathrm{~m}$, (iii) this deviation becomes noticeable only if the droplet size is less than $10^{-6}$ m. This deviation is cause by an increasing influence of the kinetic effects at the liquid-gas interface (Hertz-Knudsen-Langmuir equation) and this theory should be applied together with the diffusion equation of vapour in the air if the droplet size is less than $10^{-6} \mathrm{~m}$.

The latter conclusions show that a consideration of evaporation of microdroplets completely covered by the surface forces action (that is less than $10^{-7} \mathrm{~m}$ ) should include both deviation of the saturated vapour pressure caused by the droplet curvature and the kinetic effects.

The latent heat of vaporization results in a temperature decrease at the surface of the droplet. Due to that, the evaporation rate is reduced. This effect is more pronounced in the case of diffusion limited 
evaporation $\left(L>10^{-5} \mathrm{~m}\right)$, when vapour pressure at the droplet's surface is saturated and determined by local temperature. The effect of Marangoni convection in water droplets is negligible for droplets of size $L<10^{-5} \mathrm{~m}$. For the system considered above, Stefan flow effect appeared to be weaker than the effect of thermal Marangoni convection for $L>10^{-4} \mathrm{~m}$, but stronger for $L<10^{-4} \mathrm{~m}$. However, in all cases its influence is small and can be neglected. According to Fig. 5 the influence of latent heat of vaporization on the kinetics of evaporation is less than $5 \%$.

The presented model can be applied for evaporation of any other pure simple liquids not water only.

\section{Acknowledgments}

This work has been done under the umbrella of COST Action MP1106. Research was supported by the European Union under Grant MULTIFLOW FP7-ITN-2008-214919, and EPSRC UK grant EP/D077869/1. The work of R.G. Rubio was supported in part by the Spanish Ministerio de Ciencia e Innovación through grant FIS2009-14008-C02-01, and by ESA through project MAP-AO-00-052. Both V.M. Starov and R.G. Rubio recognise a support from European Space Agency (PASTA project).

\section{References}

1. C. Sodtke, P. Stephan, International Journal of Heat and Mass Transfer 50, 4089 (2007).

2. W.-L. Cheng, F.-Y. Han, Q.-N. Liu, R. Zhao, H.-L. Fan, Energy 36, 249 (2011).

3. Du Peng, Li Luhai, Zhao Wen, Leng Xian, Hu Xuwei, Advanced Materials Research 174, 358 (2011).

4. P.G. Campbell, L.E. Weiss, Expert Opin. Biol. Ther. 7(8), 1123 (2007).

5. S.B. Fuller, E.J. Wilhelm, J.M. Jacobson, Journal of Microelectromechanical Systems 11, 54 (2002). 
6. T. Haschke, W. Wiechert, K. Graf, E. Bonaccurso, G. Li, F.T. Suttmeier, Nanoscale and Microscale Thermophysical Engineering 11, 31 (2007).

7. R. Pericet-Camara, E. Bonaccurso, K. Graf, ChemPhysChem 9, 1738 (2008).

8. S. Karlsson, A. Rasmuson, I.N. Björn, S. Schantz, Powder Technology 207, 245 (2011).

9. J.H. Kim, W.-X. Shi, R.G. Larson, Langmuir 23, 755 (2007).

10. R.D. Deegan, O. Bakajin, T.F. Dupont, G. Huber, S.R. Nagel, T.A. Witten, Phys. Rev. E 62, 756 (2000).

11. G. Guena, C. Poulard, M. Voue, J.D. Coninck, A.M. Cazabat, Colloids Surf. A 291, 191 (2006).

12. F. Girard, M. Antoni, K. Sefiane, Langmuir 24, 9207 (2008).

13. H. Hu, R.G. Larson, J. Phys. Chem. B 110, 7090 (2006).

14. K. Sefiane, S.K. Wilson, S.David, G.J. Dunn, B.R. Duffy, Physics of Fluids 21, 062101 (2009).

15. W.D. Ristenpart, P.G. Kim, C. Domingues, J. Wan, H.A. Stone, Phys. Rev. Lett. 99, 234502 (2007).

16. R. Bhardwaj, X. Fang, D. Attinger, New J. Phys. 11, 075020 (2009).

17. S. David, K. Sefiane, L. Tadrist, Colloids Surf. A: Physicochem. Eng. Aspects 298, 108 (2007).

18. S. Semenov, V.M. Starov, R.G. Rubio, H. Agogo, M.G. Velarde, Colloids and Surfaces A: Physicochem. Eng. Aspects 391, 135 (2011).

19. S. Moosman, G.M. Homsy, Journal of Colloid and Interface Science 73, 212 (1980).

20. V. Starov, M. Velarde, C. Radke. "Dynamics of wetting and spreading”. Taylor\&Frances, in “Surfactant Sciences Series” v.138 (2007). 
21. V.S. Ajaev, T. Gambaryan-Roisman, P. Stephan, Journal of Colloid and Interface Science 342, 550 (2010).

22. A.P. Kryukov, V.Yu. Levashov, S.S. Sazhin, International Journal of Heat and Mass Transfer 47, 2541 (2004).

23. S.S. Sazhin, I.N. Shishkova, A.P. Kryukov, V.Yu. Levashov, M.R. Heikal, International Journal of Heat and Mass Transfer 50, 2675 (2007).

24. S. Semenov, V.M. Starov, R.G. Rubio, M.G. Velarde, Colloids and Surfaces A: Physicochem. Eng. Aspects 372, 127 (2010).

25. R. Krahl, M. Adamov, M.L. Avilés, E. Bänsch, “A model for two phase flow with evaporation”. Weierstraß-Institut für Angewandte Analysis und Stochastik (WIAS), Preprint ISSN 0946-8633, No 899 (2004).

26. K.P. Galvin, Chem. Eng. Sci. 60, 4659 (2005).

27. P.H. Bligh, R. Haywood, Eur. J. Phys. 7, 245 (1986). 